\title{
On the set of periods of the 2-periodic Lyness' Equation
}

Guy Bastien, Víctor Mañosa, Marc Rogalski

\begin{abstract}
We study the periodic solutions of the non-autonomous periodic Lyness' recurrence $u_{n+2}=\left(a_{n}+u_{n+1}\right) / u_{n}$, where $\left\{a_{n}\right\}_{n}$ is a cycle with positive values $a, b$ and with positive initial conditions. Among other methodological issues we give an outline of the proof of the following results: (1) If $(a, b) \neq(1,1)$, then there exists a value $p_{0}(a, b)$ such that for any $p>p_{0}(a, b)$ there exist continua of initial conditions giving rise to $2 p$-periodic sequences. (2) The set of minimal periods arising when $(a, b) \in(0, \infty)^{2}$ and positive initial conditions are considered, contains all the even numbers except $4,6,8,12$ and 20. If $a \neq b$, then it does not appear any odd period, except 1 .
\end{abstract}

\section{Introduction}

\subsection{The set of periods}

In this note we give a description of the set of periods of the 2-periodic Lyness' equations

$$
u_{n+2}=\frac{a_{n}+u_{n+1}}{u_{n}}, \quad \text { with } \quad a_{n}=\left\{\begin{array}{l}
a \text { for } n=2 \ell+1 \\
b \text { for } n=2 \ell
\end{array}\right.
$$

Guy Bastien

Institut Mathématique de Jussieu, Université Paris 6 and CNRS, France. e-mail: bastien@math.jussieu.fr

Víctor Mañosa

Departament de Matemàtica Aplicada III; Control, Dynamics and Applications Group, Universitat Politècnica de Catalunya, Spain. e-mail: victor.manosa@upc.edu

Marc Rogalski

Laboratoire Paul Painlevé, Université de Lille 1; Université Paris 6 and CNRS, France. e-mail: marc.rogalski@upmc.fr 
where $\left(u_{1}, u_{2}\right) \in \mathscr{Q}^{+}:=\left\{(x, y) \in \mathbb{R}^{2}: x>0, y>0\right\} ; \ell \in \mathbb{N}$ and $a>0, b>0$. We will only outline the main results and focus on some methodological issues. We refer the reader to [1], where a further analysis and the proofs of the main results can be found.

Theorem 1 ([1, Corollary 3]). Consider the 2-periodic Lyness' recurrence (1) for $a>0, b>0$ and positive initial conditions $u_{1}$ and $u_{2}$.

(i) If $(a, b) \neq(1,1)$, then there exists a number $p_{0}(a, b) \in \mathbb{N}$ such that for any $p>p_{0}(a, b)$ there exist continua of initial conditions giving rise to $2 p$-periodic sequences.

(ii) The set of minimal periods arising when $(a, b) \in(0, \infty)^{2}$ and positive initial conditions are considered contains all the even numbers except $4,6,8,12$ and 20 . If $a \neq b$, then it does not appear any odd period, except 1 .

As we will see, the number $p_{0}(a, b)$ is computable with our tools, in an open and dense set of the parameter space.

\subsection{A dynamical system approach}

To study the recurrence (1) we will adopt a dynamical systems approach, by using the associated composition map:

$$
F_{b, a}(x, y):=\left(F_{b} \circ F_{a}\right)(x, y)=\left(\frac{a+y}{x}, \frac{a+b x+y}{x y}\right),
$$

being $F_{a}$ and $F_{b}$ the Lyness maps $F_{\alpha}(x, y)=(y,(\alpha+y) / x)$, associated to the autonomous lyness recurrence $u_{n+2}=\left(\alpha+u_{n+1}\right) / u_{n}$.

It is very easy to observe that the first component of the iterates of the composition map gives the odd terms of the recurrence (1), while the second one gives the even terms.

The maps $F_{b, a}$ are particular cases of the family of $Q R T$ maps, which is a big class of mappings introduced in [13,14], that contains many families of discrete dynamical systems arising in mathematical physics. The QRT one constitutes a paradigmatic family of discrete integrable maps, and the reader is referred to exhaustive monograph [8] for a deeper approach.

The main feature of QRT maps is that they are defined by a geometrical construction, in such a way they preserve a fibration of the plane given by some algebraic curves of a prescribed form. In the case of the map $F_{b, a}$, this preserved fibration is given by the family of cubics:

$$
\mathscr{C}_{h}:=\{(b x+a)(a y+b)(a x+b y+a b)-h x y=0\} .
$$

Of course, this is equivalent to the fact that the map possess a first integral (invariant function) given by 


$$
V_{b, a}(x, y):=\frac{(b x+a)(a y+b)(a x+b y+a b)}{x y} .
$$

Recall that $V$ is a first integral of a map $F$ if and only if $V(F)=V$, so that the orbits of the dynamical system described by $F$ lie on the level sets of the function $V$ (the energy levels from now on). The expression of $V_{b, a}$ was also found, in another context, in [10] by following a technique for finding invariants for recurrences with periodic coefficients introduced in [9].

Since our approach depends strongly on the fact that the maps $F_{b, a}$ are QRT, it is worth noticing that the only $k$-periodic Lyness' difference equations such that their associated composition maps are QRT for all values of the parameters are the cases $k \in\{1,2,3,6\}$ (see [7, Corollary 4]).

It is known that if $a, b>0$, then any map $F_{b, a}$ has a unique fixed point $\left(x_{c}, y_{c}\right) \in$ $\mathscr{Q}^{+}$given by the solution of the system

$$
\left\{x^{2}=a+y, \quad y^{2}=b+x,\right.
$$

which corresponds to the unique global minimum of $V_{b, a}$ in $\mathscr{Q}^{+}$.

Setting $h_{c}:=\left\{V_{b, a}\left(x_{c}, y_{c}\right)\right\}$, the level sets $\mathscr{C}_{h}^{+}:=\left\{\left\{V_{b, a}=h\right\} \cap \mathscr{Q}^{+}\right\}$are given by the curves

$$
\mathscr{C}_{h}^{+}:=\{(b x+a)(a y+b)(a x+b y+a b)-h x y=0\} \cap \mathscr{Q}^{+} \text {for } h>h_{c},
$$

which are closed ones ([6, Theorem 2]; see also Figure 1).

It is also known (see [6], and also [1]) that the dynamics of $F_{b, a}$ restricted on each connected component of the invariant curves $\mathscr{C}_{h}$ is conjugate to a rotation on the unit circle.

Coming back to the problem of determining the periodic orbits of Equation (1), and since the first components of the iterates of the map $F_{b, a}$ give the odd terms of the recurrence and the second one gives the even terms, in fact, by previously proving that if $a \neq b$ there are no periodic orbits with odd period different from 1 ([1, Lemma 22]), we obtain that Theorem 1 follows as a corollary of the next result:

Theorem 2 ([1, Theorem 2]). Consider the family of maps $F_{b, a}$ given in (2) for $a, b>0$.

(i) If $(a, b) \neq(1,1)$, then there exists a value $p_{0}(a, b) \in \mathbb{N}$ such that for any $p>$ $p_{0}(a, b)$ there exist at least a continuum of initial conditions in $\mathscr{Q}^{+}\left(\right.$an oval $\left.\mathscr{C}_{h}^{+}\right)$ giving rise to $p$-periodic orbits of $F_{b, a}$.

(ii) The set of periods arising in the family $\left\{F_{b, a}, a>0, b>0\right\}$ restricted to $\mathscr{Q}^{+}$ contains all minimal periods except 2, 3, 4, 6 and 10.

It is interesting to notice that the minimal periods 2 and 3 do not appear for any $a$ and $b$ in the whole domain of definition of the dynamical system defined by $F_{b, a}$, but periods 4,6 , and 10 appear for some $a, b>0$ and some initial conditions outside $\mathscr{Q}^{+}$, see the example of Section 3.1 for instance. 


\section{Searching for periods}

In this section we explain the strategy to compute the allowed periods for a particular map $F_{b, a}$, and finally for the whole family $\left\{F_{b, a}, a, b>0\right\}$.

\subsection{Each map $F_{b, a}$ has an infinite number of periods}

In this section we give an sketch of the proof of Theorem 2 (i). First observe that since $F_{b, a}$ is conjugated to a rotation on each set $\mathscr{C}_{h}^{+}$, we can consider the rotation number function parameterized by the energy level $h \geq h_{c}: \theta_{b, a}(h)$, see Figure 1.
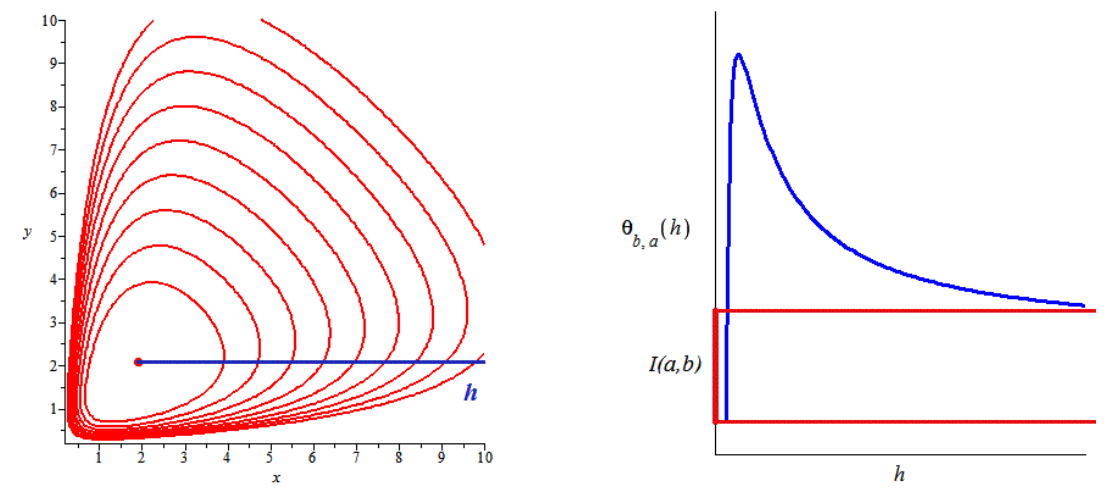

Fig. 1 The invariant curves $\mathscr{C}_{h}^{+}$, and the sketch of the graph a possible function $\theta_{b, a}(h)$ with the definition of the interval $I(a, b)$.

In [6] (and also in [1] using another approach) is proved that $\theta_{b, a}(h)$ is a continuous function in $\left[h_{c}, \infty\right)$, in fact analytic in $\left(h_{c}, \infty\right)$. Furthermore, we can compute $\theta_{b, a}\left(h_{c}\right):=\lim _{h \rightarrow h_{c}} \theta_{b, a}(h)$ and $\lim _{h \rightarrow+\infty} \theta_{b, a}(h)$, resulting that generically (that is, in an open and dense subset of the parameter space) $\theta_{b, a}\left(h_{c}\right) \neq \lim _{h \rightarrow+\infty} \theta_{b, a}(h)$. This means that generically there exists a non-degenerate interval containing some allowed rotation numbers $I(a, b)=\left\langle\theta_{b, a}\left(h_{c}\right), 2 / 5\right\rangle \subseteq \theta_{b, a}\left(h_{c},+\infty\right)$ (see Corollary 1), which means that the problem of partially knowing the set of periods of the map is equivalent to the problem of determining the set of denominators of the irreducible fractions in the interval $I(a, b)$. In summary:

Proposition 1 ([1, Proposition 13]). The rotation number map $\theta_{b, a}(h)$ is continuous in $\left[h_{c},+\infty\right)$. Furthermore

$\lim _{h \rightarrow h_{c}^{+}} \theta_{b, a}(h)=\sigma(a, b):=\frac{1}{2 \pi} \arccos \left(\frac{1}{2}\left[-2+\frac{1}{x_{c} y_{c}}\right]\right) \quad$ and $\lim _{h \rightarrow+\infty} \theta_{b, a}(h)=\frac{2}{5}$. 
As a consequence of the above result we have the following corollary:

Corollary 1. Set $I(a, b):=\left\langle\sigma(a, b), \frac{2}{5}\right\rangle:=(\min (\sigma(a, b), 2 / 5), \max (\sigma(a, b), 2 / 5))$.

(i) If $\sigma(a, b) \neq 2 / 5$, then for all $\theta \in I(a, b)$, there exists an oval $\mathscr{C}_{h}^{+}$such that $F_{b, a}\left(\mathscr{C}_{h}^{+}\right)$is conjugate to a rotation, with a rotation number $\theta_{b, a}(h)=\theta$.

(ii) For all irreducible $q / p \in I(a, b)$, there exists periodic orbits of $F_{b, a}$ of minimal period $p$.

The above result guarantees that if the parameters $a$ and $b$ are such that $\sigma(a, b) \neq$ $2 / 5$, then there exists a known non-degenerate interval containing the information of some allowed periods. The existence of this interval ensures the existence of a computable number $p_{0}(a, b) \in \mathbb{N}$, such that for all natural number $p>p_{0}(a, b)$ there exists an irreducible fraction $q / p \in I(a, b) \subseteq \theta_{b, a}\left(h_{c},+\infty\right)$, and hence proving Theorem 2 (i) for this case. The computability of $p_{0}(a, b)$ is explained in the next subsection, now we focus on the fact that the property $\sigma(a, b) \neq 2 / 5$ is a generic one. Indeed, consider the parameter space $\mathscr{P}:=\{(a, b), a, b>0\}$, then the subset $\{\sigma(a, b)=2 / 5\} \subset \mathscr{P}$ for $a, b>0$ is given by the curve

$\Gamma:=\{\sigma(a, b)=2 / 5, a, b>0\}=\left\{(a, b)=\left(\frac{t^{3}-\phi^{2}}{t}, \frac{\phi^{4}-t^{3}}{t^{2}}\right), t \in\left(\phi^{\frac{2}{3}}, \phi^{\frac{4}{3}}\right)\right\}$

see [1, Corollary 19] and Figure 2.

Fig. 2 The curve $\Gamma=$ $\{\sigma(a, b)=2 / 5$ for $a, b>0\}$

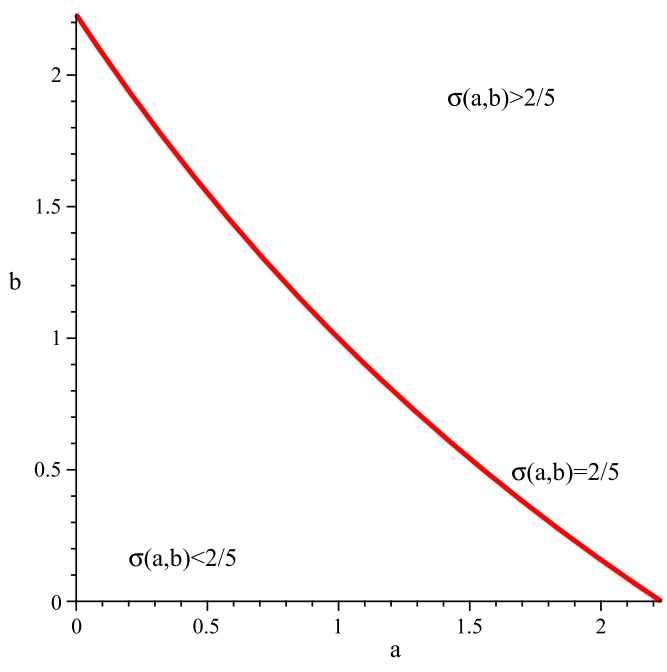
in the parameter space $\mathscr{P}$.

Of course $\mathscr{P} \backslash \Gamma$ is open and dense in $\mathscr{P}$, and therefore the fact that $I(a, b) \neq \emptyset$ and the computability of $p_{0}(a, b)$ are generic properties. 
Suppose that $\sigma(a, b)=2 / 5$. As mentioned before (see also [1, Lemma 22]) it is proved that if $a \neq b$ there are no period orbits of odd period, and that $(a, b)=$ $(1,1)$ is the only point in the parameter space satisfying both that $\sigma(a, b)=2 / 5$ and $a=b$. Then, if $(a, b) \neq(1,1)$ it is clear that $J(a, b):=\theta_{b, a}\left(\left[h_{c},+\infty\right]\right)$ is a closed interval with nonempty interior (i.e. not the single value $2 / 5$ ), since otherwise there will be globally 5-periodic maps $F_{b, a}$ with $a \neq b$, a contradiction. This last fact, implies the existence of the number $p_{0}(a, b) \in \mathbb{N}$, such that for all natural number $p>p_{0}(a, b)$ there exists an irreducible fraction $q / p \in \theta_{b, a}\left(h_{c},+\infty\right)$. This number is non-computable with our method.

Collecting all the above considerations one gets the proof of Theorem 2 (i), and hence Corollary 1 (i).

\subsubsection{Determining the particular periods of a given map $F_{b, a}$}

In this section we briefly outline a methodology for determining the set of periods of a map $F_{b, a}$, for some given fixed values $a, b>0$, in the case $\sigma \neq 2 / 5$. In this case it is clear that to determine which are the periods of a particular $F_{b, a}$ is equivalent to determine which are the irreducible fractions in $I(a, b)$, and this can be done following the next steps:

1. If $\sigma(a, b) \neq 2 / 5$, since the interval $I(a, b)$ is known, it is possible to obtain constructively a value $p$ such that for any $r>p$, there exists an irreducible fraction $q / r \in I(a, b)$. This can be done in many ways, for instance, by using the following result

Lemma 1 ([5, Theorem 25 and Corollary 26]). Consider an open interval $(c, d)$ with $0 \leq c<d$; denote by $p_{1}=2, p_{2}=3, p_{3}, \ldots, p_{n}, \ldots$ the set of all the prime numbers, ordered following the usual order. Also consider the following natural numbers:

- Let $p_{m+1}$ be the smallest prime number satisfying that $p_{m+1}>\max (3 /(d-$ c), 2),

- Given any prime number $p_{n}, 1 \leq n \leq m$, let $s_{n}$ be the smallest natural number such that $p_{n}^{s_{n}}>4 /(d-c)$.

- Set $p:=p_{1}^{s_{1}-1} p_{2}^{s_{2}-1} \cdots p_{m}^{s_{m}-1}$.

Then, for any $r>p$ there exists an irreducible fraction $q / r$ such that $q / r \in(c, d)$.

2. Finally, a finite checking (that can be done with a computer using integer arithmetics, if the above value $p$ is large) determines which values of $r \leq p$ are such that there exists an irreducible fraction $q / r \in I(a, b)$. The minimum value is then $p_{0}(a, b)$.

It is known that the rotation function $\theta_{b, a}(h)$ is not always monotonic, hence $\overline{I(a, b)} \subset \theta_{b, a}\left(\left[h_{c},+\infty\right]\right)$. So still the finite set of forbidden periods have to be determined to know exactly the set of periods of the map $F_{b, a}$. To do this, it is useful the algebraic geometric approach described in Section 3.1. 


\subsection{The periods of the family $\left\{F_{b, a}, a, b>0\right\}$}

Now we give an sketch of the proof of Theorem 2 (ii). By using the characterization of the interval $I(a, b)$ for the subfamily determined by $a=b^{2}$, it is straightforward to obtain that

$$
\bigcup_{b>0} I\left(b^{2}, b\right)=\left(\frac{1}{3}, \frac{1}{2}\right) \backslash\left\{\frac{2}{5}\right\},
$$

see [1, Lemma 16 and Proposition 17]. Hence, since

$$
\bigcup_{b>0} I\left(b^{2}, b\right)=\left(\frac{1}{3}, \frac{1}{2}\right) \backslash\left\{\frac{2}{5}\right\} \subseteq \bigcup_{a>0, b>0} I(a, b) \subseteq \bigcup_{a>0, b>0} \theta_{b, a}\left(h_{c},+\infty\right),
$$

and the map $F_{1,1}$ is globally 5-periodic (with $\theta_{1,1} \equiv 2 / 5$ ), we have the following result:

Proposition 2. For each $\theta$ in $(1 / 3,1 / 2)$ there exists $a, b>0$ and at least an oval $\mathscr{C}_{h}^{+}$, such that $F_{b, a}\left(\mathscr{C}_{h}^{+}\right)$is conjugate to a rotation with rotation number $\theta_{b, a}(h)=\theta$. In particular, for all irreducible $q / p \in(1 / 3,1 / 2)$, there exists p-periodic orbits of $F_{b, a}$.

We will be able, then, to characterize some periods of $\left\{F_{b, a}, a, b>0\right\}$ (in fact all of them), if we are able to characterize the irreducible fractions in $(1 / 3,1 / 2)$. To this end we apply Lemma 1 to the interval $(1 / 3,1 / 2)$, obtaining that for all $r \in \mathbb{N}$, such that $r>p$ with $p:=2^{4} \cdot 3^{3} \cdot 5 \cdot 7 \cdot 11 \cdot 13 \cdot 17=12252240$, there exists an irreducible fraction $q / r \in(1 / 3,1 / 2)$. Again, a finite checking determines which values of $r \leq p$ are such that $q / r \in(1 / 3,1 / 2)$, resulting that there appear irreducible fractions with all the denominators except 2,3,4,6 and 10. Hence from Proposition 2 we have that for all $p \notin\{2,3,4,6,10\}$ there exists $a, b>0$ and at least an oval $\mathscr{C}_{h}^{+}$ with associated rotation number $\theta_{b, a}(h)=q / p$, thus giving rise to a continuum (at least) of $p$-periodic orbits of $F_{b, a}$.

Notice that still it must be proved that 2, 3, 4, 6 and 10 are forbidden. The reader is referred to [1] where, to this end, it is used the algebraic geometric approach described in Section 3.1.

\section{Asymptotic behavior of $\theta_{b, a}(h)$}

The key point in all the proofs of the above results is the characterization of the intervals $I(a, b)$, and in particular the asymptotic value of $\theta_{b, a}(h)$ at infinity given in Proposition 1 (the value at the fixed point can be computed straightforwardly using tools like [4, Proposition 8], for example). In this section we sketch the proof of this result, by following the methodology introduced in [2] to study the autonomous Lyness equation. This methodology has been applied also for other equations, see $[3,4]$, for instance. 


\subsection{The map $F_{b, a}$ from an algebraic geometric approach}

In this section we will give an easy formulation of map $F_{b, a}\left(\mathscr{C}_{h}\right)$ in terms of an inner operation of the invariant curve. This description is useful to see that the action of $F_{b, a}$ on each connected component of $\mathscr{C}_{h}$ is conjugated to a rotation, and to study and locate the invariant curves filled with periodic orbits with a prescribed period, as well.

We start by extending the curves $\mathscr{C}_{h}$ and the map $F_{b, a}$ to the complex projective space. Indeed, The curves $\mathscr{C}_{h}$, in homogeneous coordinates $[x: y: t] \in \mathbb{C} P^{2}$, are

$$
\widetilde{\mathscr{C}}_{h}=\{(b x+a t)(a y+b t)(a x+b y+a b t)-h x y t=0\} .
$$

Observe that there exists three points at the infinity line: $H=[1: 0: 0] ; V=[0: 1: 0]$; and $D=[b:-a: 0]$ which are common to all the curves (see Figure 3 ).

Fig. 3 A typical curve $\tilde{\mathscr{C}}_{h}$ with $h>h_{c}$ in the real affine plane. The infinity points $V$, $H$, and $D$, are common to all curves. Observe that in the projective plane the three real unbounded components form

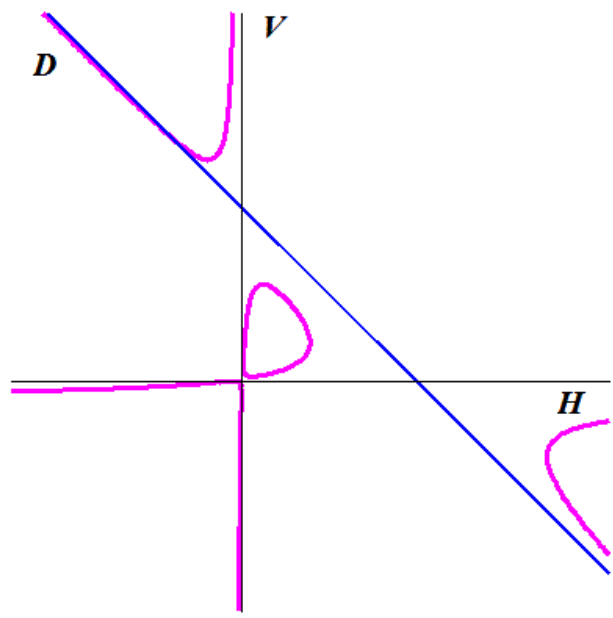
a closed curve.

The key point in our approach is the following result:

Proposition 3 ([1, Proposition 4]). If $a>0$ and $b>0$, then for all $h>h_{c}$, the curves $\widetilde{\mathscr{C}}_{h}$ are elliptic.

Recall that a cubic curve is elliptic if and only if it does not contain any singular point. Also recall that in any elliptic curve an inner sum can be defined, endowing it with a group structure. This inner operation is given by the chord-tangent process, described bellow (see [16], for instance, for further details): 
The chord-tangent law in a non-singular cubic. Consider a nonsingular cubic $\mathscr{C}$. Take two points $P$ and $Q \in \mathscr{C}$.

1. Select a point $\mathscr{O}$ on the curve, to be the neutral element of the inner sum.

2. Take the chord passing through $P$ and $Q$ (the tangent line if $P=Q$ ). It will always intersect $\mathscr{C}$ at a third point denoted by $P * Q$.

3. The point $P+Q$ is then defined as $\mathscr{O} *(P * Q)$.

The curve endowed with this operation, denoted by $(E,+, \mathscr{O})$, is an abelian group.

Fig. 4 The chord-tangent law defined on an elliptic curve, taking the infinite vertical point $V$ as a neutral element. The chord passing through $P$ and $Q$ intersects the curve at a third point denoted by $P * Q$. The point $P+Q$ is then defined as $V *(P * Q)$.

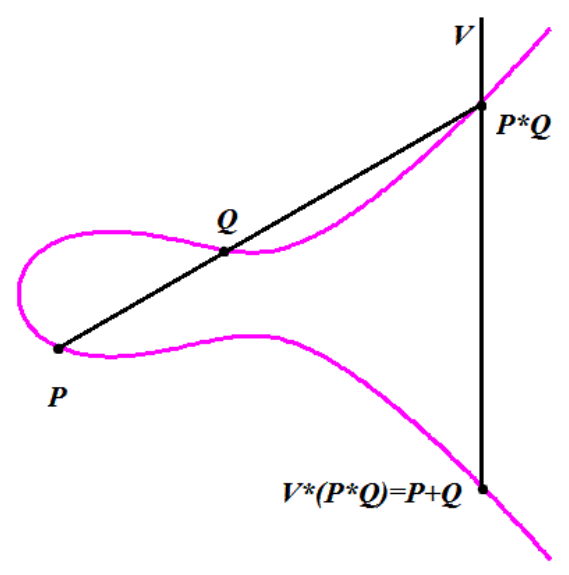

Any map $F_{b, a}$ extends to $\mathbb{C} P^{2}$ as the polynomial map: $\widetilde{F}_{b, a}([x: y: t])=\left[a y t+y^{2}\right.$ : $\left.a t^{2}+b x t+y t: x y\right]$. As for any QRT map, in fact for any birational map preserving an elliptic curve, there is a relation between the dynamics of the map and the group structure of the curve, see [11, Theorem 3]. In our case it is very easy to verify the following result (see Figure 5):

Proposition 4. For each value $h$ such that $\widetilde{\mathscr{C}}_{h}$ is elliptic,

$$
\widetilde{F}_{b,\left.a\right|_{\widetilde{\mathscr{C}}_{h}}}(P)=P+H
$$

Where + is the addition of the group law of $\widetilde{\mathscr{C}}_{h}$ taking the infinite point $V$ as the neutral element.

As a corollary of this result the action of $F_{b, a}$ on each connected component of $\mathscr{C}_{h}^{+}$is conjugated to a rotation. 
Fig. 5 The relation between the dynamics of $\widetilde{F}_{b, a}$ and the group structure of $\widetilde{\mathscr{C}}_{h}$, which also follows from the fact that $F_{b, a}$ is a QRT map.

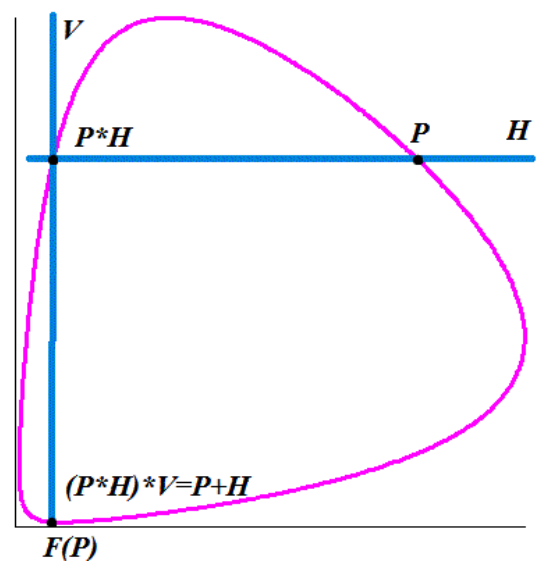

Observe that, as a consequence of the above result

$$
\widetilde{F}_{b, a}^{n}(P)=P+n H .
$$

Hence, the set $\widetilde{\mathscr{C}}_{h}$ is full of $p$-periodic orbits if and only if the following equation is satisfied

$$
p H=V,
$$

that is, if and only if $H$ is a torsion point of $\left(\tilde{\mathscr{C}}_{h},+, V\right)$. This equation is very useful to characterize the level sets where the periodic points are located, as well to characterize the forbidden periods of a particular $F_{b, a}$, or the whole family as well, as it is shown in the following example.

Example. Here we will characterize which are the level sets $\widetilde{\mathscr{C}}_{h}$, where the 4periodic orbits of $\widetilde{F}_{b, a}$ are located, by using Equation (4).

First, we notice that since the neutral element $V$ is not an inflection point of $\widetilde{\mathscr{C}}_{h}$, then the usual collinearity relation writes $A+B+C=V * V$, instead of $A+B+C=$ $V$. In consequence for any given a point $P$ on $\widetilde{\mathscr{C}}_{h}$, we have $-P=P *(V * V)=$ $P *[-a / b: 0: 1]$, see [1, Remark 7].

Now, observe that condition $4 H=V$ is equivalent to $2 H=-2 H$, and then $2 H=$ $2 H *[-a / b: 0: 1]$. An straightforward computation gives that $2 H=[0:-a: 1]$ and

$$
2 H *[-a / b: 0: 1]=\left[-\frac{a^{3}-a^{2} b^{2}-a b-h+b^{3}}{a b\left(a-b^{2}\right)},-\frac{a b+h-b^{3}}{a\left(a-b^{2}\right)}: 1\right],
$$

hence there will be 4-periodic orbits if and only if $a=\left(a b+h-b^{3}\right) /\left(a\left(a-b^{2}\right)\right)$, and then the energy level where the 4-periodic orbits are located is given by: $h_{4}:=$ $\left(a-b^{2}\right)\left(a^{2}-b\right)$. In [1, Proposition 21], it is proved that when $a, b>0$, then $h_{4}<h_{c}$, and therefore there are not 4 periodic orbits in $\mathscr{Q}^{+}$. 


\subsection{The Weierstrass form of the curves $\widetilde{\mathscr{C}}_{h}$ and the conjugated dynamics on them}

To compute the asymptotic behavior of $\theta_{b, a}(h)$ at infinity, we follow the scheme introduced in [2]. According to this, instead of looking for a normal form of the map $\widetilde{F}_{b, a}$ we look for a normal form of the invariant curve $\widetilde{\mathscr{C}}_{h}$. The idea is to find a birational change of variables preserving the group structure (a group's isomorphism) sending each curve $\left(\widetilde{\mathscr{C}}_{h},+, V\right)$ to the corresponding Weierstrass Normal Form $\left(\widehat{\mathscr{E}}_{L},+, V\right)$, where

$$
\widehat{\mathscr{E}}_{L}:=\left\{y^{2} t=4 x^{3}-g_{2} x t^{2}-g_{3} t^{3}\right\}
$$

being $g_{i}:=g_{i}(a, b, h)$, some functions whose expression can be found in [1, Proposition 11]; and where the group law is the chord-tangent one with the vertical point $V$ as a neutral element. This isomorphism, can be found in [1, Section 2.2.1] ${ }^{1}$, and it gives a conjugation between the action of $\widetilde{F}_{b, a}\left(\widetilde{\mathscr{C}}_{h}\right)$ and the action of certain birational map $\widehat{G}\left(\mathscr{E}_{L}\right)$ :

$$
\begin{aligned}
i: & \stackrel{\cong}{\left(\widetilde{\mathscr{C}}_{h},+, V\right)} \stackrel{\cong}{\rightarrow}\left(\widehat{\mathscr{E}}_{L},+, V\right) \\
\left.\widetilde{F}_{b, a}\right|_{\widetilde{\mathscr{C}}_{h}}: P \mapsto P+H & \left.\rightarrow \widehat{G}\right|_{\mathscr{E}_{L}}: P \mapsto P+\widehat{H}
\end{aligned}
$$

where $\widehat{H}=i(H)$, (see [1, Proposition 11 (iii)]). The purpose of working with the Weierstrass normal form is the following:

1. We can parameterize the Weierstrass curve by using the Weierstrass $\wp$ function.

2. This parametrization allows us to obtain the integral expression (8) for the rotation number function.

3. The asymptotic behavior of the integral expression (8) can be studied, using the tools in [2], giving the desired result.

By Proposition 3 , the cubic curves $\widetilde{\mathscr{C}}_{h}$ with $h>h_{c}$ are elliptic (thus non-singular), and therefore the curves $\widehat{\mathscr{E}}_{L}$ are also elliptic. In this case, the degree-genus formula for complex algebraic curves [12],

$$
g=\frac{1}{2}(d-1)(d-2)
$$

(where $d$ and $g$ stand for the degree and genus of the curve, respectively), gives $g=$ 1 , and hence each curve $\widehat{\mathscr{E}}_{L}$ is (holomorphic) homeomorphic to a two dimensional torus $\mathbb{T}^{2}$. It is well known (the reader is addressed to any standard book of Algebraic Geometry like [12] or [15] for instance) that there is a suitable parametrization of the curve $\widehat{\mathscr{E}}_{L}$ obtained in the following way:

\footnotetext{
${ }^{1}$ The Weierstrass Normal Form can be computed using computer algebra systems like Magma or Sage, see the Appendix. In [1] the isomorphism is explicitly constructed in order to use them in some steps of the proof of Proposition 11.
} 
The standard parametrization of the Weierstrass Cubic. For a given Weierstrass cubic $\widehat{\mathscr{E}}_{L}$, there exists a lattice

$$
\Lambda=\left\{2 n \omega_{1}+2 m i \omega_{2} \text { such that }(n, m) \in \mathbb{Z}^{2}\right\} \subset \mathbb{C},
$$

such that the map

$$
\begin{aligned}
\phi: \mathbb{T}^{2} \cong \mathbb{C} / \Lambda & \longrightarrow \widehat{\mathscr{E}}_{L} \\
z & \longrightarrow\left\{\begin{array}{ccc}
{\left[\wp(z): \wp^{\prime}(z): 1\right] \text { if }} & z \notin \Lambda, \\
V=[0: 1: 0] & \text { if } & z \in \Lambda,
\end{array}\right.
\end{aligned}
$$

where $\wp(z)$ is the Weierstrass $\wp$ function relative to $\Lambda$

$$
\wp(z)=\frac{1}{z^{2}}+\sum_{\lambda \in \Lambda \backslash\{0\}}\left[\frac{1}{(z-\lambda)^{2}}-\frac{1}{\lambda^{2}}\right],
$$

is an holomorphic homeomorphism, and it gives a parametrization of $\widehat{\mathscr{E}}_{L}$. Observe that, in consequence, the function $\wp$ satisfies the differential equation

$$
\wp^{\prime}(z)^{2}=4 \wp(z)^{3}-g_{2} \wp(z)-g_{3} .
$$

In our case $\omega_{1}$ and $\omega_{2}$ depend on $a, b$ and $h$, and the lattice $\Lambda$ can be chosen in such a way that the associated parametrization posses the following properties that are interesting for our purposes, see Figure 6:

1. The circle given by $\left[0,2 \omega_{1}\right] / \Lambda$ is projected bijectively into the real unbounded branch of the Weierstrass curve $\widehat{\mathscr{E}}_{L}$, namely $\mathscr{E}_{L}=\left\{y^{2}=4 x^{3}-g_{2} x-g_{3}\right\}$, (from now on we drop the hat when referring to the curve in the real plane).

2. The interval $\left[0, \omega_{1}\right] \in \Lambda$ is projected bijectively into the real unbounded half branch of $\mathscr{E}_{L}$ with negative coordinates.

3. The point $\omega_{1} \in \mathbb{C} / \Lambda$ is projected to the real affine point $\left(e_{1}, 0\right)$ which is the intersection of the real unbounded branch of $\mathscr{E}_{L}$, with the real abscissa axis (see Figure 6) so

$$
e_{1}=\wp\left(\omega_{1}\right)
$$

With all the above geometric consideration, we have that by direct integration of the differential equation (5) on $[0, u)$ with $u \leq \omega_{1}$, we have that in real variables

$$
u=\int_{\wp(u)}^{+\infty} \frac{\mathrm{d} s}{\sqrt{4 s^{3}-g_{2} s-g_{3}}} .
$$

On the other hand the map $\widehat{G}_{\left.\right|_{\delta_{L}}}$ has the following properties:

1. The map $\widehat{G}_{\left.\right|_{\mathscr{E}_{L}}}$ is conjugated to rotation with rotation number $\Theta(L) \in\left[0, \frac{1}{2}\right)$. 


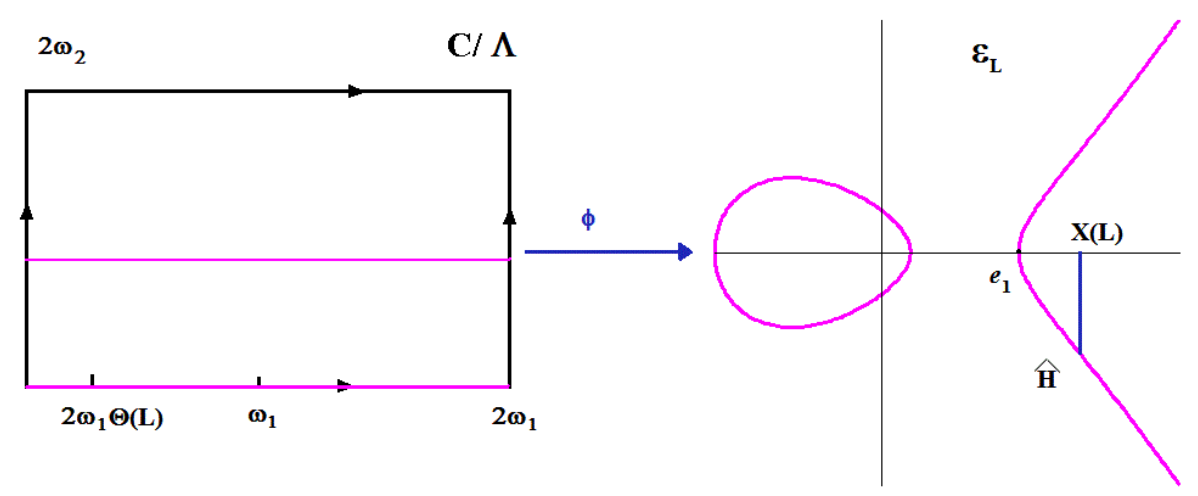

Fig. 6 Relationship between the geometry of the Weierstrass elliptic curve $\mathscr{E}_{L}$ and the action of $\widehat{G}$.

2. Since $V$ is the neutral element of $\left(\mathscr{E}_{L},+, V\right)$, then

$$
\widehat{G}_{\left.\right|_{\mathscr{E}_{L}}}(V)=V+\widehat{H}=\widehat{H}
$$

3. The point $\widehat{H}=i(H)$, is located on the real unbounded half branch of $\mathscr{E}_{L}$ with negative coordinates, [1, Proposition 11 (iii)].

In summary, $\widehat{G}$ maps the point $V$, with associated parameter $u=0$ in the interval $\left[0, \omega_{1}\right] / \Lambda$, to the point $\widehat{H}$ with some parameter $u=2 \omega_{1} \Theta(L) \in\left[0, \omega_{1}\right]$. Hence, the abscissa of $\widehat{H}$, say $X(L)$, must satisfy $X(L)=\wp\left(2 \omega_{1} \Theta(L)\right)$. Using the integral expression (7) we have that

$$
2 \omega_{1} \Theta(L)=\int_{X(L)}^{+\infty} \frac{\mathrm{d} s}{\sqrt{4 s^{3}-g_{2} s-g_{3}}} .
$$

Now, using Equation (6) and again (7), we get the desired integral expression for the rotation number

$$
\Theta(L)=\frac{1}{2} \frac{\int_{X(L)}^{+\infty} \frac{\mathrm{d} s}{\sqrt{4 s^{3}-g_{2} s-g_{3}}}}{\int_{e_{1}}^{+\infty} \frac{\mathrm{d} s}{\sqrt{4 s^{3}-g_{2} s-g_{3}}}} .
$$

The asymptotic analysis of this equation can be done using the tools developed in [2], resulting that $\Theta(L) \sim 2 / 5$. To this end, the asymptotic expansions of some of the elements of the curve and of $X(L)$ must to be known, and this is one of the main computational obstruction in the proof, see [1] for further details.

Acknowledgements The second author is partially supported by Spain's Ministry of Science and Technology (MCYT) through grant DPI2011-25822. CoDALab group is supported by Generalitat de Catalunya through the SGR program. 


\section{Appendix}

The following, is a Magma code for computing the Weierstrass Normal form $\widehat{\mathscr{E}}_{L}$ of the curves $\widetilde{\mathscr{C}}_{h}$.

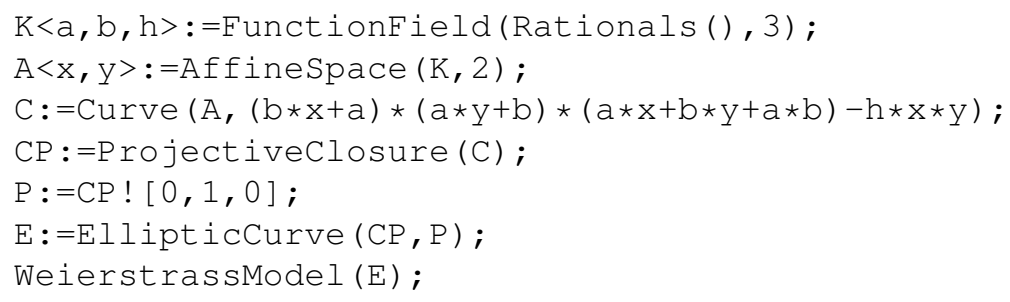

It can be processed in the Magma's site http://magma.maths.usyd.edu.au/calc/. Using Magma V2.18-8, it gives the expression of $\widehat{\mathscr{E}}_{L}$ in 0.220 seconds with a total memory usage of $12.06 \mathrm{MB}$.

\section{References}

1. Bastien G., Mañosa V., Rogalski M.: On periodic solutions of 2-periodic Lyness difference equations. arXiv:1201.1027v2 [math.DS] (2012).

2. Bastien G., Rogalski M.: Global behavior of the solutions of Lyness' difference equation $u_{n+2} u_{n}=u_{n+1}+a$, J. Difference Equations and Appl. 10, 977-1003 (2004).

3. Bastien G., Rogalski M.: On the Algebraic Difference Equation $u_{n+2} u_{n}=\psi\left(u_{n+1}\right)$ in $\mathbb{R}_{*}^{+}$ Related to a Family of Elliptic Quartics in the Plane, Advances in Difference Equations 3, 227-261 (2005).

4. Bastien G., Rogalski M.: On algebraic difference equations $u_{n+2}+u_{n}=\psi\left(u_{n+1}\right)$ in $\mathbf{R}$ related to a family of elliptic quartics in the plane, J. Math. Anal. Appl. 326, 822-844 (2007).

5. Cima A., Gasull A., Mañosa V.: Dynamics of the third order Lyness difference equation, J. Difference Equations and Appl. 13, 855-884 (2007).

6. Cima A., Gasull A., Mañosa V.: On 2- and 3- periodic Lyness difference equations, J. Difference Equations and Appl. 18, 849-864 (2012).

7. Cima A., Gasull A., Mañosa V.: Integrability and non-integrability of periodic nonautonomous Lyness recurrences. arXiv:1012.4925v2 [math.DS] (2011)

8. Duistermaat, J.J.: Discrete Integrable Systems: QRT Maps and Elliptic Surfaces. Springer, New York (2010).

9. Feuer J., Janowski E.J., Ladas G.: Invariants for some rational recursive sequence with periodic coefficients, J. Difference Equations and Appl. 2, 167-174 (1996).

10. Janowski E.J., Kulenović M.R.S., Nurkanović Z.: Stability of the $k$ th order Lyness' equation with period- $k$ coefficient, Int. J. Bifurcations \& Chaos 17, 143-152 (2007).

11. Jogia D., Roberts J. A. G., Vivaldi F.: An algebraic geometric approach to integrable maps of the plane, J. Physics A: Mathematical \& General 39, 1133-1149 (2006).

12. Kirwan, F.: Complex Algebraic Curves. Cambridge University Press, Cambridge (1992).

13. Quispel G.R.W., Roberts J.A.G., Thompson C.J.: Integrable mappings and soliton equations, Phys. Lett. A 126 419-421 (1988).

14. Quispel G.R.W., Roberts J.A.G., Thompson C.J.: Integrable mappings and soliton equations II, Physica D 34 183-192 (1989).

15. Silverman, J.: The arithmetic of elliptic curves. Springer, New York (2009).

16. Silverman J., Tate J.: Rational points on elliptic curves. Springer, New York (1992). 